\title{
NON-COMMUTATIVE BOUNDED VILENKIN SYSTEMS
}

\author{
P. G. DODDS and F. A. SUKOCHEV
}

\begin{abstract}
We consider orthonormal systems in spaces of measurable operators associated with a finite von Neumann algebra which contain the classical bounded Vilenkin systems. We show that they form Schauder bases in all reflexive non-commutative $L_{p}$-spaces when taken in the lexicographic order. This is a non-commutative analogue of a theorem of Paley.
\end{abstract}

\section{Introduction}

The principal theme of this paper finds its source in the classical theorems of Paley [Pa]. In the terminology of the theory of Banach spaces, these theorems assert that the classical Walsh system, taken in the Walsh-Paley ordering (respectively, partitioned into dyadic blocks) forms a (Schauder) basis (respectively, an unconditional decomposition) in each of the spaces $L_{p}[0,1], 1<p<\infty$. The classical Walsh system may be identified with the dual group of the familiar dyadic group. From this point of view, the notion of a Vilenkin system introduced in [Vi] generalizes that of the Walsh system. Watari [Wa] extended Paley's theorems to bounded Vilenkin systems and showed that such systems when enumerated lexicographically not only form a Schauder basis in each of the reflexive $L_{p}$-spaces, but also permit a finer unconditional decomposition than that given by the dyadic decomposition associated with the Walsh system.

The study of orthogonal series in the setting of non-commutative $L_{p}$ spaces was initiated in [SF1,2]. In this setting, the classical Walsh system is replaced by a system of eigenoperators arising from the action of a compact Abelian group on the underlying von Neumann algebra. The methods of [SF1,2] are based on the fact that non-commutative $L_{p}$-spaces have the unconditionality property for martingale difference sequences and this approach permits an extension of Paley's theorems to the non-commutative setting. Our intention in this paper is to study orthogonal systems in spaces of measurable operators affiliated with a finite von Neumann algebra, which

\footnotetext{
* Research supported by A.R.C.

Received December 1, 1997; in revised form November 21, 1998.
} 
contain the classical bounded Vilenkin systems as a special case. We will show (Theorem 2.4) that such systems when enumerated lexicographically form Schauder bases in all reflexive (non-commutative) $L_{p}$-spaces and that their "fine" partitions yield unconditional decompositions (Theorem 2.3) of these spaces. While our results contain those of [Pa, Wa] and [SF 1,2] as special cases, the methods and approach here are quite different and are adapted to exhibiting the finer features of the unconditional structure, which reflect the classical results of Watari in the commutative setting.

\section{Preliminaries}

A compact topological group $G$ is called a Vilenkin group if it is totally disconnected, commutative and its topology satisfies the second axiom of countability. An important example of a Vilenkin group is the group $G_{\mathbf{m}}=\prod_{n=0}^{\infty} \mathbf{Z}_{m(n)}$ where $\mathbf{m}=\{m(n) \mid n \in \mathbf{N} \cup\{0\}\}$ is a sequence of natural numbers greater than one and $Z_{m(n)}$ is the discrete cyclic group of order $m(n)$. The Vilenkin system $\left\{\psi_{n}\right\}_{n=0}^{\infty}$ (introduced by N.Ya. Vilenkin [Vi]) is the set $\hat{G}_{\mathbf{m}}=\left(\coprod_{n=0}^{\infty} \hat{\mathbf{Z}}_{m(n)}=\coprod_{n=0}^{\infty} \mathbf{Z}_{m(n)}\right)$ enumerated lexicographically via

$$
\psi_{n}:=\prod_{k=0}^{\infty} r_{k}^{n_{k}} \quad n \in \mathrm{N}
$$

where $r_{k}$ is given by

$$
r_{k}(x):=\exp \left(2 \pi i \frac{x_{k}}{m(k)}\right)
$$

for $x=\left(x_{0}, x_{1}, \ldots\right) \in G_{\mathbf{m}}$ and the sequence $\left\{n_{k}\right\}_{k=0}^{\infty}$ is defined by

$$
n=\sum_{k=0}^{\infty} n_{k} M_{k}, \quad 0 \leq n_{k}<m(k)
$$

where $M_{0}:=1$ and $M_{k}:=m(k-1) M_{k-1}$. There exists a well-known identification between $G_{\mathbf{m}}$ equipped with Haar measure $m$ and the interval $(0,1)$ with Lebesgue measure. This identification allows us to consider the Vilenkin system $\left\{\psi_{n}\right\}_{n=0}^{\infty}$ as a complete multiplicative orthonormal system in $L_{2}(0,1)$. In the simplest case when $m(k)=2, k \in \mathrm{N} \cup\{0\}$, the group $G_{\mathbf{m}}$ is the dyadic group $\mathrm{D}$, the system $\left\{r_{k}\right\}_{k \in \mathrm{N} \cup\{0\}}$ may be identified with the usual Rademacher system $\left\{\rho_{k}\right\}_{k=0}^{\infty}$ where

$$
\rho_{k}(x)=(-1)^{x_{k}}=\left\{\begin{aligned}
1 & \text { if } x_{k}=0 \\
-1 & \text { if } x_{k}=1 .
\end{aligned}\right.
$$

for $x=\left\{x_{n}\right\}_{n=0}^{\infty} \in \mathrm{D}$ and $k \in \mathrm{N} \cup\{0\}$. In this case, the Vilenkin system coincides with the familiar Walsh system $\left\{w_{n}\right\}_{n=0}^{\infty}$ taken in the Walsh-Paley enu- 
meration [SWS]. For harmonic analysis on compact Vilenkin groups, we refer the reader to $[\mathrm{Vi}],[\mathrm{AVDR}],[\mathrm{BaR}],[\mathrm{SWS}]$ and references contained therein.

Let $X$ be a Banach space and let $\left\{X_{i}\right\}_{i=1}^{\infty}$ denote a sequence of closed subspaces of $X$ such that

$$
X_{i} \cap X_{j}=\{0\}, i \neq j .
$$

If, for each $x \in X$, there exists a unique sequence $\left\{x_{i}\right\}_{i=1}^{\infty}, x_{i} \in X_{i}$ such that

$$
x=\sum_{i=1}^{\infty} x_{i}
$$

then $\left\{X_{i}\right\}_{i=1}^{\infty}$ is said to be a (Schauder) decomposition of $X$. If $\operatorname{dim} X_{i}=1, i \in \mathrm{N}, x_{i} \in X_{i}, x_{i} \neq 0$, then the sequence $\left\{x_{i}\right\}_{i=1}^{\infty}$ is called a (Schauder) basis of $X$. If, for any $x=\sum_{i=1}^{\infty} x_{i} \in X$ and any sequence $\epsilon_{i}= \pm 1, i=1,2, \ldots$, the series

$$
\sum_{i=1}^{\infty} \epsilon_{i} x_{i}
$$

converges in $X$ then the sequence $\left\{X_{i}\right\}_{i=1}^{\infty}$ is said to form an unconditional decomposition of $X$.

It was shown by Paley [Pa] that the Walsh-Paley system $\left\{w_{n}\right\}_{n=0}^{\infty}$ forms a Schauder basis in each of the spaces $L_{p}(0,1), 1<p<\infty$, thereby complementing the corresponding theorem of Riesz for the trigonometric system. Paley showed, in fact, that the system of subspaces $\left\{\Delta_{n}\right\}_{n=0}^{\infty} \cup w_{0}$ where $\left.\Delta_{l}:=\operatorname{clm}\left\{\prod_{k=0}^{l} \rho_{k}^{n_{k}}: n_{l}=1\right\}=\operatorname{clm}\left\{w_{i}: 2^{l} \leq i \leq 2^{l+1}-1\right)\right\}, \quad l=0,1,2, \ldots$ forms an unconditional decomposition of $L_{p}(0,1), 1<p<\infty$. Here, $\operatorname{clm}(A)$ denotes the closed linear manifold generated by $A$.

The same decomposition may be introduced for any Vilenkin group $G_{\mathrm{m}}$ : it is formed by martingale differences with respect to the sequence of $\sigma$-subalgebras $\left\{\mathscr{F}_{k}\right\}_{k=0}^{\infty}$ where $\mathscr{F}_{k}$ is generated by the cosets of

$$
\bar{G}_{k}:=\prod_{n=0}^{k-1}\{0\} \times \prod_{n=k}^{\infty} \mathbf{Z}_{m(n)}, \quad\left(\bar{G}_{0}:=G_{\mathbf{m}}\right)
$$

and hence generates an unconditional decomposition of $L_{p}(0,1), 1<p<\infty$. See, for instance, [LT 2] 2.c.4, 2.c.5 and the subsequent discussion and references. Nevertheless, the latter decomposition is not sufficiently fine to obtain the complete analogue of Paley's result for an arbitrary Vilenkin 
system which is bounded in the sense that $\sup _{n>0} m(n)<\infty$. In fact, Watari [Wa] considered the system $\delta:=\left\{\delta_{n, j}\right\}_{n=0, j=1}^{\infty, m(n)-1} \cup \psi_{0}$ where for fixed $l \in \mathrm{N} \cup\{0\}, 0 \leq j \leq m(n)-1$,

$$
\delta_{l, j}:=\operatorname{clm}\left\{\prod_{k=0}^{l} r_{k}^{n_{k}}: n_{l}=j\right\}=\operatorname{clm}\left\{\psi_{i}: j M_{l} \leq i \leq(j+1) M_{l}-1\right\}
$$

and showed that this finer decomposition still yields an unconditional decomposition of $L_{p}(0,1), 1<p<\infty$ ([Wa] Theorem 1$)$.

The prime objective of this paper is to show that Watari's results continue to hold in non-commutative spaces. To introduce the setting, we need some additional notation and terminology.

For standard results from von Neumann algebra theory, we refer to [BR], [Di], [Pe], [Sa] and [Ta].

Let $\mathscr{M}$ be a finite von Neumann algebra with a faithful, finite, normal trace $\tau$ and unit $\mathbf{1}$, acting in separable Hilbert space. Let $\alpha=\left\{\alpha_{g}\right\}_{g \in G_{\mathrm{m}}}$ be a $G_{\mathrm{m}}$-flow on $\mathscr{M}$, i.e. $\alpha$ is an ergodic, ultraweakly continuous representation of $G_{\mathrm{m}}$ by $*$-automorphisms of $\mathscr{M}$ such that for any $g \in G_{\mathrm{m}}$, we have $\tau \circ \alpha_{g}=\tau$. Given the character $\gamma$ from the dual group $\hat{G}_{\mathbf{m}}$, any operator $0 \neq W_{\gamma} \in \mathscr{M}$ is called an eigenoperator for $\alpha$ corresponding to the eigenvalue $\gamma$ whenever

$$
\alpha_{g}\left(W_{\gamma}\right)=\langle\gamma, g\rangle W_{\gamma}, \quad \forall g \in G_{\mathbf{m}} .
$$

Since $\alpha$ is ergodic, the operator $W_{0}$ may be taken to be $\mathbf{1}$. In addition, since $\alpha$ is faithful, each eigenspace is the one-dimensional span of some unitary operator and the weak closure of the linear span of the eigenspaces is $\mathscr{M}$ itself. See, for example, [St] Lemma 2.1 and [OPT] section 2.3. Further, for each $\gamma \in \hat{G}_{\mathbf{m}}$, there exists a unimodular number $\beta(\gamma)$ such that

$$
W_{\gamma}^{*} W_{-\gamma}=\beta(\gamma) \mathbf{1}
$$

and for all $\gamma, \lambda \in \hat{G}_{\mathbf{m}}$, there exist unimodular numbers $\alpha(\gamma, \lambda), \alpha^{\prime}(\gamma, \lambda)$ such that

$$
W_{\gamma} W_{\lambda}=\alpha(\gamma, \lambda) W_{\lambda} W_{\gamma}, \quad W_{\gamma} W_{\lambda}=\alpha^{\prime}(\gamma, \lambda) W_{\gamma+\lambda} .
$$

For $n \in \mathrm{N}$, we will denote $W_{\psi_{n}}$ by $W_{n}$. Combining (1.1) with (1.6), it follows further that for any eigenoperator $W_{l}$ there exists a unimodular number $\alpha(l)$ such that

$$
W_{l}=\alpha(l) \prod_{k=0}^{\infty} W_{r_{k}{ }_{k}}
$$

where (see (1.3)) 


$$
l=\sum_{k=0}^{\infty} n_{k} M_{k}, \quad 0 \leq n_{k}<m(k) .
$$

It will be convenient to introduce the following terminology. We adhere to the notation of the previous paragraph.

Definition 1.1. If $\alpha=\left\{\alpha_{g}\right\}_{g \in G_{\mathrm{m}}}$ is a $G_{\mathrm{m}}$-flow on $\mathscr{M}$, then a Vilenkin system $\mathscr{V}=\left\{W_{n}\right\}_{n=0}^{\infty}=\left\{W_{\psi_{n}}\right\}_{n=0}^{\infty}$ is a complete system $\left\{W_{\gamma}: \gamma \in \hat{G}_{\mathbf{m}}\right\}$ of unitary eigenoperators enumerated by the lexicographic ordering of $\hat{G}_{\mathrm{m}}$. In the special case that $G_{\mathbf{m}}=\mathrm{D}$, then any Vilenkin system $\left\{W_{n}\right\}_{n=0}^{\infty}$ will be called a Walsh-Paley system. Further, if

$$
A=\sup _{n \geq 0} m(n)<\infty,
$$

then the Vilenkin system $\mathscr{V}$ is called bounded.

Note that, if $m$ is Haar measure on $G_{\mathbf{m}}$, if $(\mathscr{M}, \tau)$ is the von Neumann algebra $L_{\infty}\left(G_{\mathbf{m}}, m\right)$ with trace given by integration and if $\alpha$ is induced by forward translation then the Vilenkin system $\left\{W_{n}\right\}_{n=0}^{\infty} \subseteq L_{\infty}\left(G_{\mathbf{m}}, m\right)$ may be identified with the classical Vilenkin system $\left\{\psi_{n}\right\}_{n=0}^{\infty}$ in the sense that for every $n \in \mathrm{N} \cup\{0\}$ there exists a unimodular number $\alpha_{n}$ such that $W_{n}=\alpha_{n} \psi_{n}$.

For $1 \leq p<\infty$, the $L_{p}$-space associated with $(\mathscr{M}, \tau)$ is given by

$$
L_{p}(\mathscr{M}, \tau):=\left\{x \in \tilde{\mathscr{M}} \mid\|x\|_{L_{p}(\mathscr{M}, \tau)}:=\left(\tau\left(|x|^{p}\right)\right)^{\frac{1}{p}}<\infty\right\}
$$

where $\tilde{\mathscr{M}}$ is the space of all $\tau$-measurable operators affiliated with $\mathscr{M}$ (see [Se], $[\mathrm{FK}])$. Non-commutative $L_{p}$-spaces are special cases of the non-commutative symmetric spaces $E(\mathscr{M}, \tau)$ associated with $(\mathscr{M}, \tau)$ and the symmetric function space $E[0, \tau(\mathbf{1}))$ ([LT 2], [KPS]), where $\mathbf{1}$ is the identity in $\mathscr{M}$. In the present setting, we shall consider only those spaces $E(\mathscr{M}, \tau)$ which are separable interpolation spaces for some couple $\left(L_{p}(\mathscr{M}, \tau), L_{q}(\mathscr{M}, \tau)\right)$ with $1<p \leq q<\infty$. This is the case, for example if the space $E[0, \tau(\mathbf{1}))$ is itself separable and has non-trivial Boyd indices (see [LT2]). For more detailed information concerning the spaces $E(\mathscr{M}, \tau)$, we refer to [DDP1,2], [DS], [SC], [SF1] and the references contained therein.

Since the vector space spanned by the set of all eigenoperators $\left\{W_{\gamma}\right\}_{\gamma \in \hat{G}_{\mathrm{m}}}$ is weakly dense in $\mathscr{M}$, it follows that any Vilenkin system forms a complete orthonormal system in $L_{2}(\mathscr{M}, \tau)$. In what follows, we set $G=G_{\mathrm{m}}$ for the sake of brevity. We set

$$
G_{-1}=\{0\}, \hat{G}_{-1}=\{\hat{0}\}, U_{-1}:=\hat{G}_{-1},
$$

and for any $n \in \mathrm{N} \cup\{0\}, k \in \hat{\mathbf{Z}}_{m(n)}, k \neq \hat{0}$ we define 


$$
G_{n}=\prod_{k=0}^{n} \mathbf{Z}_{m(k)} \times \prod_{k=n+1}^{\infty}\{0\}, \quad \hat{G}_{n}=\prod_{k=1}^{n} \hat{\mathbf{Z}}_{m(k)} \times \prod_{k=n+1}^{\infty}\{\hat{0}\},
$$

$$
U_{n}:=\left\{\gamma=\left(\gamma_{0}, \gamma_{1}, \ldots\right) \in \hat{G} \mid \gamma_{n} \neq \hat{0}, \gamma_{n+1}=\hat{0}, \gamma_{n+2}=\hat{0}, \ldots\right\}=\cup_{k=1}^{m(n)-1} U_{n}^{k} .
$$

It easily follows from $(1.1)-(1.3)$ that

$$
\operatorname{card} U_{n}^{k}=M_{n}
$$

for all $n \geq 0$ and $k \in \hat{\mathbf{Z}}_{m(n)}, k \neq \hat{0}$, and that

$$
\text { card } U_{n}=M_{n+1}-M_{n} \text {. }
$$

For all $n \in \mathrm{N} \cup\{0\}, j=1, m(n)-1$ we let

$$
\begin{aligned}
\delta_{n, j}:=\operatorname{clm}\left\{W_{\gamma}:\right. & \left.\gamma \in U_{n}^{j}\right\}=\operatorname{clm}\left\{W_{i}: j M_{n} \leq i \leq(j+1) M_{n}-1\right\}, \\
\Delta_{n} & =\operatorname{clm}\left\{W_{\gamma}: \gamma \in U_{n}\right\} \\
& =\operatorname{clm}\left\{W_{i}: M_{n} \leq i \leq M_{n+1}-1\right\} \\
& =\operatorname{clm}\left\{\delta_{n, j}: 1 \leq j \leq m(n)-1\right\}
\end{aligned}
$$

and

$$
\Delta_{-1}=\operatorname{clm}\left\{W_{\gamma}: \gamma \in U_{-1}\right\}=\operatorname{clm}\{\mathbf{1}\} .
$$

The first non-commutative analogues of Paley's results were given in [SF1,2].

Theorem 1.2 [SF1,2], [FS]. The sequence

$$
\Delta=\left\{\Delta_{n}\right\}_{n=-1}^{\infty}
$$

forms an unconditional decomposition of any $L_{p}(\mathscr{M}, \tau)$ provided $1<p<\infty$.

This further implies that any non-commutative Walsh-Paley system forms a Schauder basis in any $L_{p}(\mathscr{M}, \tau), 1<p<\infty$. We shall show below that the sequence

$$
\delta:=\left\{\delta_{n, j}\right\}_{n=0, j=1}^{\infty, m(n)-1} \cup \mathbf{1}
$$

also forms an unconditional decomposition of any $L_{p}(\mathscr{M}, \tau)$ provided $1<p<\infty$ and infer further that any non-commutative bounded Vilenkin system forms a Schauder basis in any $L_{p}(\mathscr{M}, \tau), 1<p<\infty$. Our methods are completely different from those employed in [Wa]. 


\section{Main results}

Our purpose in this section is to show that any bounded Vilenkin system is a Schauder basis (Theorem 2.4) and that the system $\delta$ forms an unconditional decomposition in $L_{p}(\mathscr{M}, \tau), 1<p<\infty$ (Theorem 2.3).

Following [LPP] (see also [DS], [PX]), we denote by $E(\mathscr{M}, \tau)_{R}$ (respectively, $\left.E(\mathscr{M}, \tau)_{L}\right)$, the completion of the space of finitely non-zero sequences $\left\{x_{k}\right\}_{k=1}^{N} \subseteq E(\mathscr{M}, \tau)$ equipped with the norm

$$
\left\|\left\{x_{k}\right\}_{k=1}^{N}\right\|_{E(\mathscr{M}, \tau)_{R}}:=\left\|\left(\sum_{k=1}^{N}\left|x_{k}\right|^{2}\right)^{\frac{1}{2}}\right\|_{E(\mathscr{M}, \tau)}
$$

(respectively,

$$
\left.\left\|\left\{x_{k}\right\}_{k=1}^{N}\right\|_{E(\mathscr{M}, \tau)_{L}}:=\left\|\left(\sum_{k=1}^{N}\left|x_{k}^{*}\right|^{2}\right)^{\frac{1}{2}}\right\|_{E(\mathscr{M}, \tau)}\right) .
$$

We set

$$
E(\mathscr{M}, \tau)_{R, L}:=E(\mathscr{M}, \tau)_{R}+E(\mathscr{M}, \tau)_{L},
$$

and denote by $E(\mathscr{M}, \tau)_{S}$ the completion of the space of finitely non-zero sequences $\left\{x_{k}\right\}_{k=1}^{N} \subseteq E(\mathscr{M}, \tau)$ equipped with the norm

$$
\left\|\left\{x_{k}\right\}_{k=1}^{N}\right\|_{E(\mathscr{M}, \tau)_{S}}:=\left\|\left(\sum_{k=1}^{N}\left|x_{k}\right|_{S}^{2}\right)^{\frac{1}{2}}\right\|_{E(\mathscr{M}, \tau)}
$$

where

$$
|x|_{S}^{2}=\frac{1}{2}\left(x^{*} x+x x^{*}\right) .
$$

By the symbol $\approx$ we shall denote a two-sided estimate with absolute constants (whose values may change from line to line).

It follows from [St] Lemma 2.1(4) that

$$
\mathscr{M}_{n}:=\operatorname{clm}\left\{W_{\gamma}: \gamma \in \hat{G}_{n}\right\}
$$

is a von Neumann subalgebra of $\mathscr{M}$ for all $n=-1,0,1, \ldots$ Noting that the orthogonal projection

$$
\mathscr{E}_{n}: L_{2}(\mathscr{M}, \tau) \rightarrow \mathscr{M}_{n}, \quad n=-1,0,1, \ldots
$$

is the conditional expectation of $(\mathscr{M}, \tau)$ with respect to $\mathscr{M}_{n}$, we have

$$
\Delta_{n}=\left(\mathscr{E}_{n}-\mathscr{E}_{n-1}\right)\left(L_{2}(\mathscr{M}, \tau)\right), \quad n=-1,0,1, \ldots
$$


where we set for convenience

$$
\left(\mathscr{E}_{-1}-\mathscr{E}_{-2}\right) x:=\mathscr{E}_{-1} x=\tau(x) \mathbf{1}, \quad x \in L_{1}(\mathscr{M}, \tau) .
$$

By a standard inequality which relates unconditional decompositions to Rademacher averages and using the non-commutative Khintchine inequalities proved in [LP], [LPP] Corollaries II.2, III.4, Remark III.6, Theorem 1.2 yields immediately that

$$
\begin{aligned}
\|x\|_{L_{p}(\mathscr{M}, \tau)} & \approx\left\|\left\{\left(\mathscr{E}_{n}-\mathscr{E}_{n-1}\right) x\right\}\right\|_{L_{p}(\mathscr{M}, \tau)_{R, L}}, \\
\|x\|_{L_{p}(\mathscr{M}, \tau)} & \approx\left\|\left\{\left(\mathscr{E}_{n}-\mathscr{E}_{n-1}\right) x\right\}\right\|_{L_{p}(\mathscr{M}, \tau)_{S}}
\end{aligned}
$$

for $1<p<2$ and $2<p<\infty$ respectively.

We shall need the following non-commutative version of the Stein inequality due to Pisier and $\mathrm{Xu}[\mathrm{PX}]$.

Proposition 2.1 [PX]. Define the map $Q$ on all finite sequences $a=\left\{a_{n}\right\}_{n \geq 0}$ in $L_{p}(\mathscr{M}, \tau)$ by $Q(a)=\left\{\mathscr{E}_{n-1} a_{n}\right\}_{n \geq 0}$. Then for any $1<p<\infty$, there is a positive constant $\gamma_{p}$ such that

$$
\|Q(a)\|_{L_{p}(\mathscr{M}, \tau)_{L}} \leq \gamma_{p}\|a\|_{L_{p}(\mathscr{M}, \tau)_{L}}, \quad\|Q(a)\|_{L_{p}(\mathscr{M}, \tau)_{R}} \leq \gamma_{p}\|a\|_{L_{p}(\mathscr{M}, \tau)_{R}} .
$$

REMARK 2.2. We shall apply the preceding result of Pisier and $\mathrm{Xu}$ in the case that the sequence $\left\{\mathscr{E}_{n}\right\}$ is a sequence of conditional expectations associated with a $G$-flow. In this special case, it is possible to give an alternative proof of Proposition 2.1 based on a transference method [SF1,2].

THEOREM 2.3. The system $\delta$ forms an unconditional decomposition of any $L_{p}(\mathscr{M}, \tau)$ provided $1<p<\infty$. Moreover, for $1<p<2$ (respectively, $2 \leq p<\infty)$ and any finite sequence $\left\{x_{n, j}\right\}$ with $x_{n, j} \in \delta_{n, j}$ we have

$$
\left\|\sum_{n, j} x_{n, j}\right\|_{L_{p}(\mathscr{M}, \tau)} \approx\left\|\left\{x_{n, j}\right\}\right\|_{L_{p}(\mathscr{M}, \tau)_{R, L}}
$$

(respectively,

$$
\left.\left\|\sum_{n, j} x_{n, j}\right\|_{L_{p}(\mathscr{M}, \tau)} \approx\left\|\left\{x_{n, j}\right\}\right\|_{L_{p}(\mathscr{M}, \tau)_{S}}\right) .
$$

Proof. The second assertion of Theorem 2.3 follows immediately from the first assertion combined with the results [LP], [LPP] Corollaries II.2, III.4, Remark III.6 (see also [DS] Theorem 1.1, Corollary 1.2). We shall therefore concentrate on the first assertion.

We note first that via the usual duality arguments, it suffices to consider the case $2<p<\infty$. In order to prove that the system of subspaces $\delta$ forms 
an unconditional decomposition of $L_{p}(\mathscr{M}, \tau)$ for some $p \in(2, \infty)$, it suffices to show that the norms of the (orthogonal) projections $P_{\delta^{\prime}}$ on finite subsequences $\delta^{\prime} \subseteq \delta$ are uniformly bounded in $L_{p}(\mathscr{M}, \tau)$. Since the projection $P_{\delta^{\prime}}$ for $\delta^{\prime}=\{\mathbf{1}\}$ coincides with the conditional expectation $\mathscr{E}_{-1}$, we need to consider the norms of the (orthogonal) projections

$$
P_{\mathscr{B}}: L_{p}(\mathscr{M}, \tau) \stackrel{\text { onto }}{\longrightarrow} \operatorname{clm}\left\{\delta_{n, j}:(n, j) \in \mathscr{B}\right\}
$$

defined for all finite sets $\mathscr{B}$ of indices $(n, j), n=0,1,2, \ldots$; $j=1,2, \ldots, m(n)-1$. By assumption, for any $n=0,1,2, \ldots$, the set

$$
\mathscr{B} \cap\{(n, j) \mid j=1,2, \ldots, m(n)-1\}
$$

contains no more than $A-1$ elements. It follows that there exist $r \leq A-1$ sets of indices

$$
\mathscr{B}_{1}, \mathscr{B}_{2}, \ldots, \mathscr{B}_{r}
$$

such that

$$
\cup_{i=1}^{r} \mathscr{B}_{i}=\mathscr{B}, \quad \mathscr{B}_{i} \cap \mathscr{B}_{j}=\emptyset, 1 \leq i, j \leq r, i \neq j
$$

and such that the intersection

$$
\mathscr{B}_{i} \cap\{(n, j) \mid j=1,2, \ldots, m(n)-1\}
$$

does not contain more than one element for any $n=0,1,2, \ldots$ Assume for a moment that there exists a positive constant $K$ such that

$$
\left\|P_{\mathscr{B}_{i}}\right\|_{L_{p}(\mathscr{M}, \tau) \rightarrow L_{p}(\mathscr{M}, \tau)} \leq K, \quad 1 \leq i \leq r .
$$

Since

$$
\sum_{i=1}^{r} P_{\mathscr{B}_{i}}=P_{\mathscr{B}}
$$

for $1 \leq i \leq r$, we obtain from (2.7) that

$$
\left\|P_{\mathscr{B}}\right\|_{L_{p}(\mathscr{M}, \tau) \rightarrow L_{p}(\mathscr{M}, \tau)} \leq K(A-1)
$$

whence

$$
\left\|P_{\delta^{\prime}}\right\|_{L_{p}(\mathscr{M}, \tau) \rightarrow L_{p}(\mathscr{M}, \tau)} \leq K(A-1)+1
$$

for any finite subsequence $\delta^{\prime} \subseteq \delta$. Consequently, the estimate (2.7) will suffice to complete the proof of Theorem 2.3. From now till the end of the proof, let

$$
\mathscr{B}_{I}=\left\{\left(n, j_{n}\right) \mid n \in I, \quad 1 \leq j_{n} \leq m(n)-1\right\}
$$


where $I$ is a finite set of non-negative integers. Reformulating (2.7), we have to prove that there exists a constant $K$ such that, if $P_{\mathscr{B}_{I}}$ denotes the (orthogonal) projection

$$
P_{\mathscr{B}_{I}}: L_{p}(\mathscr{M}, \tau) \stackrel{\text { onto }}{\longrightarrow} \operatorname{clm}\left\{\delta_{n, j_{n}}: n \in I\right\}
$$

we have

$$
\sup _{I}\left\|P_{\mathscr{B}_{I}}\right\|_{L_{p}(\mathscr{M}, \tau) \rightarrow L_{p}(\mathscr{M}, \tau)} \leq K
$$

Letting

$$
N=\max \{n \mid n \in I\}
$$

and taking into account that $\left\|\mathscr{E}_{N}\right\|_{L_{p}(\mathscr{M}, \tau) \rightarrow L_{p}(\mathscr{M}, \tau)} \leq 1$, we may rewrite (2.8) as

$$
\left\|\sum_{n \in I} \sum_{\gamma \in U_{n}^{j n}} c_{\gamma} W_{\gamma}\right\|_{L_{p}(\mathscr{M}, \tau)} \leq K\left\|\sum_{\gamma \in \hat{G}_{N}} c_{\gamma} W_{\gamma}\right\|_{L_{p}(\mathscr{M}, \tau)}
$$

where $\left\{c_{\gamma}\right\}_{\gamma \in \hat{G}_{N}}$ is an arbitrary family of scalars. We set for brevity

$$
x:=\sum_{\gamma \in \hat{G}_{N}} c_{\gamma} W_{\gamma}, \quad x=\sum_{n} x_{n}=\sum_{n, j} x_{n, j},
$$

where

$$
x_{n}:=\sum_{\gamma \in U_{n}} c_{\gamma} W_{\gamma}, \quad x_{n, j}:=\sum_{\gamma \in U_{n}^{j}} c_{\gamma} W_{\gamma}\left(=P_{n, j} x\right)
$$

Claim. For any $n \in I$, we have

$$
W_{r_{n}^{j_{n}}} \mathscr{E}_{n-1}\left(W_{r_{n}^{j_{n}}}^{*} x_{n}\right)=\overline{\beta\left(r_{n}^{j_{n}}\right)} x_{n, j_{n}}, \quad \mathscr{E}_{n-1}\left(x_{n} W_{r_{n}^{j_{n}}}^{*}\right) W_{r_{n}^{j_{n}}}=\beta\left(r_{n}^{j_{n}}\right) x_{n, j_{n}}
$$

where $\beta\left(r_{n}^{j_{n}}\right)$ is the scalar given by (1.5).

Proof of the Claim. We have

$$
x_{n}=\sum_{j=1}^{m(n)-1} x_{n, j} .
$$

Each element $x_{n, j}$ from (2.11) may be written as a linear combination of elements $\prod_{k=0}^{n} W_{r_{k}}$ where $0 \leq l_{k} \leq m(k)-1$ and $l_{n}=j$ (see (2.10), (1.7) and (1.8)). The latter fact together with (2.5) and (1.6) imply that $W_{r_{n}^{n}}^{*} x_{n, j}$ may be written as a linear combination of elements $\prod_{k=0}^{n} W_{r_{k}^{\prime}}$ where $l_{k}^{\prime}=l_{k}$, 
$0 \leq k \leq n-1$ and $l_{n}^{\prime}=l_{n}-j_{n}$ (subtraction in $\mathbf{Z}_{m(n)}$ ). Thus, $l_{n}^{\prime}=0$ if and only if $l_{n}=j_{n}$, i.e. if and only if $j=j_{n}$. It follows immediately that

$$
\mathscr{E}_{n-1}\left(W_{r_{n}^{\prime}}^{*} x_{n, j}\right)= \begin{cases}0 & \text { if } j \neq j_{n}, \\ W_{r_{n}^{j_{n}}}^{*} x_{n, j} & \text { if } j=j_{n}\end{cases}
$$

The first equality of the claim follows immediately from (2.12) and (1.5) and the second may be established similarly. This completes the proof of the claim.

We may now complete the proof of Theorem 2.3 as follows. From the claim and from Proposition 2.1 we have

$$
\begin{aligned}
\left\|\left\{x_{n, j_{n}}\right\}_{n \in I}\right\|_{L_{p}(\mathscr{M}, \tau)_{R}} & =\left\|\left\{\mathscr{E}_{n-1}\left(W_{r_{n}}^{*} x_{n}\right)\right\}_{n \in I}\right\|_{L_{p}(\mathscr{M}, \tau)_{R}} \\
& \leq \gamma_{p}\left\|\left\{W_{r_{n}^{\prime}}^{*} x_{n}\right\}_{n \in I}\right\|_{L_{p}(\mathscr{M}, \tau)_{R}} \\
& =\gamma_{p}\left\|\left\{x_{n}\right\}_{n \in I}\right\|_{L_{p}(\mathscr{M}, \tau)_{R}}
\end{aligned}
$$

and

$$
\begin{aligned}
\left\|\left\{x_{n, j_{n}}\right\}_{n \in I}\right\|_{L_{p}(\mathscr{M}, \tau)_{L}} & =\left\|\left\{\mathscr{E}_{n-1}\left(x_{n} W_{r_{n}^{j_{n}}}^{*}\right)\right\}_{n \in I}\right\|_{L_{p}(\mathscr{M}, \tau)_{L}} \\
& \leq \gamma_{p}\left\|\left\{x_{n} W_{r_{n}^{j_{n}}}^{*}\right\}_{n \in I}\right\|_{L_{p}(\mathscr{M}, \tau)_{L}} \\
& =\gamma_{p}\left\|\left\{x_{n}\right\}_{n \in I}\right\|_{L_{p}(\mathscr{M}, \tau)_{L}},
\end{aligned}
$$

whence

$$
\left\|\left\{x_{n, j_{n}}\right\}_{n \in I}\right\|_{L_{p}(\mathscr{M}, \tau)_{S}} \leq \gamma_{p}\left\|\left\{x_{n}\right\}_{n \in I}\right\|_{L_{p}(\mathscr{M}, \tau)_{S}} .
$$

Since $\left(\mathscr{E}_{n}-\mathscr{E}_{n-1}\right) x_{n, j_{n}}=x_{n, j_{n}}$ we may deduce now (2.9) from (2.1), (2.13) and again (2.1) as follows

$$
\begin{aligned}
\left\|\sum_{n \in I} \sum_{\gamma \in U_{n}^{j_{n}}} c_{\gamma} W_{\gamma}\right\|_{L_{p}(\mathscr{M}, \tau)} & =\left\|\sum_{n \in I} x_{n, j_{n}}\right\|_{L_{p}(\mathscr{M}, \tau)} \\
& \approx\left\|\left\{x_{n, j_{n}}\right\}_{n \in I}\right\|_{L_{p}(\mathscr{M}, \tau)_{S}} \\
& \leq \gamma_{p}\left\|\left\{x_{n}\right\}_{n \in I}\right\|_{L_{p}(\mathscr{M}, \tau)_{S}} \\
& \approx \gamma_{p}\|x\|_{L_{p}(\mathscr{M}, \tau)} .
\end{aligned}
$$

We remark that Theorem 2.3 does not hold for Vilenkin systems which are not bounded, even in the classical commutative setting. See, for example [Wa] Section 6 and also [BaR], [AVDR].

THEOREM 2.4. Any bounded non-commutative Vilenkin system $\mathscr{V}$ is a Schauder basis in $L_{p}(\mathscr{M}, \tau)$ provided $1<p<\infty$. 
Proof. The asertion of Theorem 2.4 will be estalished as soon as the inequality

$$
\left\|\sum_{i=0}^{l} \alpha_{i} W_{i}\right\|_{L_{p}(\mathscr{M}, \tau)} \leq C\left\|\sum_{i=0}^{s} \alpha_{i} W_{i}\right\|_{L_{p}(\mathscr{M}, \tau)}
$$

is proved for an arbitrary scalars $\alpha_{0}, \ldots, \alpha_{s}$ and some positive constant $C$ which depends only on $p$ and does not depend on the particular choice of scalars $\alpha_{i}$ and integers $l, s$ with $l \leq s$ (see [LT 1]). We shall show that (2.14) holds with the constant $C=K(A-1)+1$, with $K$ given as in (2.7). Recall (see (1.7)) that for any given positive integer $l$ we have

$$
W_{l}=\alpha(l) \prod_{k=0}^{\infty} W_{r_{k}^{n_{k}}}
$$

where

$$
l=\sum_{k=0}^{\infty} n_{k} M_{k}, \quad 0 \leq n_{k}<m(k) .
$$

We set

$$
L:=\prod_{k=0}^{\infty} W_{r_{k}^{m(k)-n_{k}}}
$$

where the operator $W_{r_{k}^{m(k)-n_{k}}}$ is understood to be the unit operator as soon as $n_{k}=0$. Using the definition of Walsh-Paley enumeration and (2.15), (2.16), we see that for any $i \leq l$

$$
W_{i} L \in \operatorname{clm}\left\{W_{\gamma}: \gamma \in \mathbf{B}\right\}
$$

where

$$
\mathbf{B}:=\hat{G}_{-1} \cup \bigcup_{k=0}^{\infty}\left(\bigcup_{\substack{k^{\prime}=x+m(k)-n_{k} \\ x=0,1,2, \ldots, n_{k}-1 \in Z_{m(k)}}} U_{k}^{k^{\prime}}\right)
$$

and similarly, for any $i>l$ we have

$$
W_{i} L \in \operatorname{clm}\left\{W_{\gamma}: \gamma \in \mathbf{A}\right\}
$$

where 


$$
\mathbf{A}:=\bigcup_{k=0}^{\infty}\left(\bigcup_{\substack{k^{\prime}=x+m(k)-n_{k} \\ x=n_{k}+1, n_{k}+2, \ldots, m(k)-1 \in Z_{m(k)}}} U_{k}^{k^{\prime}}\right)
$$

Let

$$
\mathscr{B}:=\left\{\left(k, k^{\prime}\right) \mid U_{k}^{k^{\prime}} \in \mathbf{B}\right\}, \quad \mathscr{A}:=\left\{\left(k, k^{\prime}\right) \mid U_{k}^{k^{\prime}} \in \mathbf{A}\right\} .
$$

and let $\delta^{\prime}$ be the subsequence of $\delta$ consisting of all $\delta_{n, j}$ such that $(n, j) \in \mathscr{B}$ and $\{\mathbf{1}\}$. It follows from (2.17) and (2.18) that $\mathscr{B} \cap \mathscr{A}=\emptyset$. Consequently, from $(2.7)^{\prime}$, we have that

$$
\begin{aligned}
\left\|\sum_{i=0}^{l} \alpha_{i} W_{i}\right\|_{L_{p}(\mathscr{M}, \tau)} & =\left\|\sum_{i=0}^{l} \alpha_{i} W_{i} L\right\|_{L_{p}(\mathscr{M}, \tau)} \\
& =\left\|P_{\delta^{\prime}}\left(\sum_{i=0}^{l} \alpha_{i} W_{i} L+\sum_{i=l+1}^{s} \alpha_{i} W_{i} L\right)\right\|_{L_{p}(\mathscr{M}, \tau)} \\
& \leq(K(A-1)+1)\left\|\sum_{i=0}^{l} \alpha_{i} W_{i} L+\sum_{i=l+1}^{s} \alpha_{i} W_{i} L\right\|_{L_{p}(\mathscr{M}, \tau)} \\
& =(K(A-1)+1)\left\|\sum_{i=0}^{s} \alpha_{i} W_{i}\right\|_{L_{p}(\mathscr{M}, \tau)} .
\end{aligned}
$$

This suffices to complete the proof of the theorem.

We remark that the space $E(0,1)$ has non-trivial Boyd indices if and only if $E(0,1)$ is an interpolation space for some pair of reflexive $L_{p}$-spaces on $(0,1)$. See [LT2]. Accordingly, the following corollaries are consequences of Theorems 2.4, 2.3 via [DDP2].

Corollary 2.5. A non-commutative bounded Vilenkin system $\mathscr{V}$ forms a Schauder basis in any symmetric operator space $E(\mathscr{M}, \tau)$ associated with $(\mathscr{M}, \tau)$ and the separable symmetric function space $E(0,1)$ with non-trivial Boyd indices.

COROLlary 2.6. The system $\delta$ forms an unconditional decomposition of any symmetric operator space $E(\mathscr{M}, \tau)$ associated with $(\mathscr{M}, \tau)$ and the separable symmetric function space $E(0,1)$ with non-trivial Boyd indices. 


\section{Examples}

This section is intended to illustrate the results of the previous section in the setting of the finite hyperfinite factor $\mathscr{R}$. Non-commutative Vilenkin systems arising in this setting naturally encompass the classical ones.

Let $G$ be a compact Abelian group with Haar measure $\mu$, and suppose that $\mathscr{G}$ is a countably infinite discrete group. Let $\mathscr{A}$ be the Abelian von Neumann algebra $L_{\infty}(G, \mu)$ acting by multiplication on the Hilbert space $\mathscr{H}=L_{2}(G, \mu)$ and suppose that $\Lambda: \mathscr{G} \rightarrow \operatorname{Aut}(\mathscr{A})$ is an action of $\mathscr{G}$ on $\mathscr{A}$. The crossed product $\mathscr{R}(\mathscr{A}, \mathscr{G}, \Lambda)$ is defined as follows ([Ta] Definition V 7.4, [Sa] Chapter 4.2). We let $\mathscr{K}=L_{2}(\mathscr{G}, \mathscr{H})$ be the space of $\mathscr{H}$-valued functions $\xi(\cdot)$ on $\mathscr{G}$ for which $\sum_{t \in \mathscr{G}}\|\xi(t)\|^{2}<\infty$ and consider the covariant pair $(\pi, \lambda)$ of representations on $\mathscr{K}$ defined by setting

$$
\begin{aligned}
\pi(x) \xi(t) & :=\Lambda_{t}^{-1}(x)(\xi(t)), \quad t \in \mathscr{G}, \quad x \in \mathscr{A}, \\
\lambda_{s} \xi(t) & :=\xi(t-s), \quad s, t \in \mathscr{G},
\end{aligned}
$$

for all $\xi \in \mathscr{K}$. It follows that

$$
\operatorname{Ad} \lambda_{s} \pi(x)\left(:=\lambda_{s} \pi(x) \lambda_{s}^{*}\right)=\pi\left(\Lambda_{s}(x)\right),
$$

for all $x \in \mathscr{A}, s \in \mathscr{G}$. The crossed product $\mathscr{R}(\mathscr{A}, \mathscr{G}, \Lambda)$ is defined to be the von Neumann algebra generated by $\pi(\mathscr{A})$ and $\left\{\lambda_{t}: t \in \mathscr{G}\right\}$

We will now restrict our attention to the special setting in which $G=G_{\mathbf{m}}$ is a bounded Vilenkin group and $\mathscr{G}:=\hat{G}_{\mathrm{m}}$. It is clear that we may identify the discrete group $\mathscr{G}$ with a dense countable subgroup of $G_{\mathrm{m}}$. We let $\Lambda: \mathscr{G} \rightarrow \operatorname{Aut}(\mathscr{A})$ be given by forward translation on $\mathscr{A}=L_{\infty}\left(G_{\mathrm{m}}, \mu\right)$, that is

$$
\Lambda_{s}(x)(t)=x(t+s), \quad s \in \mathscr{G}, t \in G_{\mathbf{m}} .
$$

Without confusion, we shall also denote by $\Lambda$ the action of $G_{\mathrm{m}}$ on $\mathscr{A}$ given by forward translation. Since the action $\Lambda: \mathscr{G} \rightarrow A u t(\mathscr{A})$ is free and ergodic, it follows that the crossed product $\mathscr{R}(\mathscr{A}, \mathscr{G}, \Lambda)$ is the unique hyperfinite factor $(\mathscr{R}, \tau)$ of type $I I_{1}$.

Lemma 3.1. The action $\left\{\operatorname{Ad} \Lambda:=\operatorname{Ad} \lambda_{s}\right\}_{s \in \mathscr{G}}: \mathscr{G} \rightarrow \operatorname{Aut}(\mathscr{R})$ extends to an ultraweakly continuous representation $\alpha=\left\{\alpha_{s}\right\}_{s \in G_{\mathrm{m}}}: G_{\mathrm{m}} \rightarrow \operatorname{Aut}(\mathscr{R})$ by tracepreserving automorphisms of $\mathscr{R}$.

Proof. For each $n=1,2, \ldots$, let $\mathscr{G}_{n}:=\hat{G}_{n}$, where $G_{n}$ is as given in (1.8) and let $\mathscr{R}_{n}$ be the set of all elements $x \in \mathscr{R}$ of the form

$$
x=\sum_{t \in \mathscr{G}_{n}} \pi\left(x_{t}\right) \lambda_{t}
$$

with $x_{t} \in \mathscr{A}$ for all $t \in \mathscr{G}_{n}$. We set $\mathscr{R}_{0}:=\cup_{n=1}^{\infty} \mathscr{R}_{n}$. We note that each $\mathscr{R}_{n}$ is a 
von Neumann subalgebra of $\mathscr{R}$ and that $\mathscr{R}_{0}$ is a ${ }^{*}$-subalgebra of $\mathscr{R}$ which is dense for the $\sigma$-strong* topology.

For each $s \in G_{\mathrm{m}}$, we define $\alpha_{s}: \mathscr{R}_{0} \rightarrow \mathscr{R}_{0}$ by setting

$$
\alpha_{s}(x):=\sum_{t \in \mathscr{G}_{n}} \pi\left(\Lambda_{s}\left(x_{t}\right)\right) \lambda_{t}
$$

for each element $x=\sum_{t \in \mathscr{G}_{n}} \pi\left(x_{t}\right) \lambda_{t} \in \mathscr{R}_{n}, n=1,2, \ldots$ The restriction of $\alpha_{s}$ to each von Neumann subalgebra $\mathscr{R}_{n}$ is a ${ }^{*}$-automorphism and therefore an isometry for the operator norm. This implies that $\alpha_{s}: \mathscr{R}_{0} \rightarrow \mathscr{R}_{0}$ is a surjective isometry for the operator norm. Since $\alpha_{s}$ is a trace-preserving automorphism of $\mathscr{R}_{0}$, it follows that

$$
\tau\left(\alpha_{s}(x) y\right)=\tau\left(x \alpha_{s}^{-1}(y)\right)
$$

for all $x, y \in \mathscr{R}_{0}$. Consequently, $\alpha_{s}$ is continuous for the weak topology $\sigma\left(\mathscr{R}, \mathscr{R}_{0}\right)$ induced on $\mathscr{R}$ by $\mathscr{R}_{0}$, considered as a linear subspace of the predual $L_{1}(\mathscr{R}, \tau)$.

Let us now observe that if $\left\{y_{i}\right\}$ is any net in $\mathscr{R}$ such that $\left\{y_{i}\right\}$ converges to 0 for the $\sigma$-strong topology, then $\tau\left(y_{i} \cdot\right)$ converges to 0 uniformly on any subset of $\mathscr{R} \subseteq L_{1}(\mathscr{R}, \tau)$ which is bounded for the operator norm. In fact, there exist sequences $\left\{\xi_{n}\right\}_{n=1}^{\infty},\left\{\eta_{n}\right\}_{n=1}^{\infty} \subseteq \mathscr{K}$ such that

$$
\sum_{n=1}^{\infty}\left\|\xi_{n}\right\|^{2}<\infty, \sum_{n=1}^{\infty}\left\|\eta_{n}\right\|^{2}<\infty
$$

for which

$$
\tau(z)=\sum_{n}^{\infty}\left\langle z \xi_{n}, \eta_{n}\right\rangle, \in \mathscr{R} .
$$

From this it follows that

$$
\left|\tau\left(y_{i} y\right)\right| \leq\|y\|\left(\sum_{n=1}^{\infty}\left\|\eta_{n}\right\|^{2}\right)^{\frac{1}{2}}\left(\sum_{n=1}^{\infty}\left\|y_{i} \xi_{n}\right\|^{2}\right)^{\frac{1}{2}}
$$

for all $y \in \mathscr{R}$ and this implies the assertion. Since $\mathscr{R}_{0}$ is dense in $\mathscr{R}$ for the $\sigma$ strong topology, and since $\alpha_{s}$ is an isometry for the operator norm, it now follows that the restriction of $\alpha_{s}$ to bounded sets in $\mathscr{R}_{0}$ is continuous for the weak topology $\sigma(\mathscr{R}, \mathscr{R})$ induced on $\mathscr{R}$ by $\mathscr{R} \subseteq L_{1}(\mathscr{R}, \tau)$. Since $\mathscr{R}$ is dense in $L_{1}(\mathscr{R}, \tau)$, a simple argument shows that the restriction of $\alpha_{s}$ to bounded sets of $\mathscr{R}_{0}$ is continuous for the weak ${ }^{*}$-topology $\sigma\left(\mathscr{R}, L_{1}(\mathscr{R}, \tau)\right)$. We obtain, therefore, that the restriction of $\alpha_{s}$ to bounded subsets of $\mathscr{R}_{0}$ is ultraweakly continuous. Since every element of $\mathscr{R}$ is in the ultraweak closure of a boun- 
ded subset of $\mathscr{R}_{0}([\mathrm{Di}]$, Chapter I.3, Theorem 3$)$ and since the bounded sets of $\mathscr{R}$ are ultraweakly compact and therefore ultraweakly complete, it now follows that $\alpha_{s}$ extends to an ultraweakly continuous linear mapping of $\mathscr{R}$ which we continue to denote by $\alpha_{s}$. By ultraweak continuity, it follows easily that $\alpha_{s}=\operatorname{Ad} \lambda_{s}$ whenever $s \in \mathscr{G}$, that $\alpha_{s+t}=\alpha_{s} \alpha_{t}$ for all $s, t \in G_{\mathbf{m}}$, and that $\alpha_{s}$ is a trace preserving $*$-automorphism of $\mathscr{R}$ for all $s \in G_{\mathbf{m}}$.

Since

$$
\sigma\left(L_{\infty}\left(G_{\mathbf{m}}, \mu\right), L_{1}\left(G_{\mathbf{m}}, \mu\right)\right)-\lim _{s \rightarrow 0} \Lambda_{s}(x)=x
$$

holds for all $x \in L_{\infty}\left(G_{\mathbf{m}}, \mu\right)$, it follows that $\tau\left(\alpha_{s}(x) y\right) \rightarrow 0$ as $s \rightarrow 0$ for all $x \in \mathscr{R}_{0}, y \in \mathscr{R}$. That this assertion continues to hold for all $x \in \mathscr{R}$, $y \in L_{1}(\mathscr{R}, \tau)$ follows again from the fact that $\mathscr{R}_{0}$ is dense in $\mathscr{R}$ for the $\sigma$ strong topology, the fact that $\alpha_{s}$ is a surjective isometry for the operator norm for each $s \in G$, the density of $\mathscr{R}$ in $L_{1}(\mathscr{R}, \tau)$ and the observation in the first part of the preceding paragraph. We omit the details. It follows that $\alpha:=\left\{\alpha_{s}\right\}_{s \in G_{\mathrm{m}}}$ is an ultraweakly continuous representation of $G_{\mathrm{m}}$ by trace preserving automorphisms of $\mathscr{R}$. This suffices to complete the proof of the Lemma.

We now consider the unitary representation $u=\left\{u_{t}: t \in G_{\mathbf{m}}\right\}$ on $\mathscr{K}$ given by setting

$$
\left(u_{t} \xi\right)(s):=\overline{\langle t, s\rangle}(s) . \quad \xi \in \mathscr{K}, t \in G_{\mathbf{m}}, s \in \mathscr{G},
$$

where the group $G_{\mathrm{m}}$ is identified as the dual of the group $\mathscr{G}$. It is not difficult to check that the pair $(\lambda, u)$ satisfy the (so-called) Weyl commutation relations:

$$
u_{t} \lambda_{s}=\langle t, s\rangle \lambda_{s} u_{t}, \quad t \in G_{\mathbf{m}}, s \in \mathscr{G}
$$

From (3.3), it follows that

$$
\text { Ad } u_{t} \operatorname{Ad} \lambda_{s}=\operatorname{Ad} \lambda_{s} \text { Ad } u_{t}, \in G_{\mathbf{m}}, s \in \mathscr{G}
$$

and consequently the equality

$$
\text { Ad } u_{t} \alpha_{s}=\alpha_{s} \text { Ad } u_{t}, s, t \in G_{\mathbf{m}}
$$

follows from the ultraweak continuity of $\alpha$ and the density of $\mathscr{G}$ in $G_{\mathrm{m}}$.

Proposition 3.2. If $\beta=\left\{\beta_{(s, t)}:(s, t) \in G_{\mathbf{m}} \times G_{\mathbf{m}}\right\}$ is given by

$$
\beta_{(s, t)}:=\alpha_{t} \text { Ad } u_{s}, \quad(s, t) \in G_{\mathbf{m}} \times G_{\mathbf{m}}
$$

then $\beta$ is an ultraweakly continuous ergodic representation of $G_{\mathbf{m}} \times G_{\mathbf{m}}$ on $R$ 
by trace-preserving *automorphisms of $\mathscr{R}$. For each character $(\gamma, \nu) \in \hat{G}_{\mathbf{m}} \times \hat{G}_{\mathbf{m}}$, the eigenoperator $W_{\gamma, \nu}$ may be taken to be $\nu(\gamma) \lambda_{\nu}$.

Proof. It follows from (3.4) that $\beta$ is an ultraweakly continuous representation of $G_{\mathrm{m}} \times G_{\mathrm{m}}$ on $\mathscr{R}$ by trace-preserving automorphisms of $\mathscr{R}$. To verify the last assertion of the proposition, note first that the equality

$$
\text { Ad } u_{s}(\pi(x))=\pi(x)
$$

holds for each $s \in G_{\mathbf{m}}$ and $x \in L_{\infty}\left(G_{\mathbf{m}}, \mu\right)$. Further, if $\gamma \in \hat{G}_{\mathbf{m}}$, observe that $\Lambda_{t}(\gamma)=\langle t, \gamma\rangle \gamma$ so that

$$
\alpha_{t}(\pi(\gamma))=\pi\left(\Lambda_{t}(\gamma)\right)=\langle t, \gamma\rangle \pi(\gamma)
$$

for all $t \in G_{\mathbf{m}}$. Consequently, if $(\gamma, \nu) \in \hat{G}_{\mathbf{m}} \times \hat{G}_{\mathbf{m}}$, it follows that

$$
\begin{aligned}
\beta_{(s, t)}\left(\pi(\gamma) \lambda_{\nu}\right) & =\alpha_{t} \operatorname{Ad} u_{s}\left(\pi(\gamma) \lambda_{\nu}\right) \\
& =\alpha_{t}(\pi(\gamma)) \text { Ad } u_{s}\left(\lambda_{\nu}\right) \\
& =\langle s, \nu\rangle\langle t, \gamma\rangle \pi(\gamma) \lambda_{\nu}
\end{aligned}
$$

for all $(s, t) \in G_{\mathbf{m}} \times G_{\mathbf{m}}$ and this suffices to complete the proof of the proposition.

We note that $\pi(\gamma)=W_{(\gamma, \hat{0})}$ for each $\gamma \in \hat{G}$. Consequently the system of eigenoperators $\left\{W_{(\gamma, \nu)}\right\}_{(\gamma, \nu) \in \hat{G} \times \hat{G}}$ contains the classical Vilenkin system.

We shall identify the group $G_{\mathbf{m}} \times G_{\mathbf{m}}$ (respectively, $\hat{G}_{\mathbf{m}} \times \hat{G}_{\mathbf{m}}$ ) with the Vilenkin group $G_{2 \mathrm{~m}}$ (respectively, $\hat{G}_{2 \mathrm{~m}}$ ) where

$$
\mathbf{2 m}:=\{l(n) \mid n \in \mathrm{N} \cup\{0\}\}, \quad l(2 n)=l(2 n+1):=m(n), \quad n=0,1,2, \ldots
$$

It follows that any $\gamma=\left(\gamma_{0}, \gamma_{1}, \ldots\right) \in \hat{G}_{2 \mathrm{~m}}$ can be considered as a pair $\left(\nu_{1}, \nu_{2}\right)$ where

$$
\nu_{1}:=\left(\gamma_{2 k}\right)_{k=0}^{\infty} \quad \text { and } \quad \nu_{2}:=\left(\gamma_{2 k+1}\right)_{k=0}^{\infty}
$$

are elements of $\hat{G}_{\mathbf{m}}$.

The following is a consequence of Proposition 3.2, Theorem 2.4 and Corollary 2.5 and extends to Vilenkin systems the results of [SF1,2] concerning non-commutative Walsh systems in $\mathscr{R}$.

THEOREM 3.3. The bounded Vilenkin system $\mathscr{V}$ consisting of the eigenoperators

$$
\left\{W_{\gamma}\right\}_{\gamma \in \hat{G}_{2 \mathbf{m}}}=\left\{W_{\left(\nu_{1}, \nu_{2}\right)}\right\}_{\left(\nu_{1}, \nu_{2}\right) \in \hat{G}_{\mathbf{m}} \times \hat{G}_{\mathbf{m}}}=\left\{\pi\left(\nu_{1}\right) \lambda_{\nu_{2}}\right\}_{\left(\nu_{1}, \nu_{2}\right) \in \hat{G}_{\mathbf{m}} \times \hat{G}_{\mathbf{m}}}
$$

forms a Schauder basis in any space $L_{p}(\mathscr{R}, \tau)$, provided $1<p<\infty$. The same assertion holds in any symmetric operator space $E(\mathscr{R}, \tau)$ associated with $(\mathscr{R}, \tau)$ 
and the separable symmetric function space $E(0,1)$ with non-trivial Boyd indices.

Further insight into the system just considered may be obtained via tensor products. Let $\mathbf{M}_{m(n)}$ be the algebra of all complex $m(n) \times m(n)$ matrices with the normalized trace $t r_{m(n)}$ and the identity $\mathbf{1}_{m(n)}$. We let $e_{i j}^{m(n)}, i, j \in \mathbf{Z}_{m(n)}$ be the usual system of matrix units in $\mathbf{M}_{m(n)}$. Let $\mathbf{M}_{m(n)}^{d}$ be the subalgebra of $\mathbf{M}_{m(n)}$ generated by all $e_{i i}^{m(n)}, i \in \mathbf{Z}_{m(n)}$. Via the same arguments as in [Sa] p. 206 , there exists a $*$-isomorphism $\vartheta$ between $\mathscr{R}=\mathscr{R}\left(L_{\infty}\left(G_{2 \mathrm{~m}}, \mu\right), \hat{G}_{2 \mathrm{~m}}, \Lambda\right)$ and the infinite tensor-product

$$
\otimes_{n=0}^{\infty}\left(\mathbf{M}_{m(n)}, t r_{m(n)}\right)
$$

of $\mathbf{M}_{m(n)}$ along the traces $t r_{m(n)}$ (see, for example, [Str] A.17, [CO] pp. 62-65, [Pe] pp. 206, 282-283).

In particular, $\vartheta \circ \pi$ is a $*$-isomorphism between the algebras $L_{\infty}\left(G_{\mathbf{m}}, \mu\right)$ and $\left(\mathscr{R}^{d}, \tau\right)$ where $\mathscr{R}^{d}$ is the commutative von Neumann subalgebra of $\mathscr{R}$ defined as the (so)-closure of the linear span of the set

$$
\begin{gathered}
\mathscr{R}^{d}:= \\
\left\{x=x_{0} \otimes x_{1} \otimes \ldots \otimes x_{l} \otimes \mathbf{1}_{m(l+1)} \otimes \mathbf{1}_{m(l+2)} \ldots, \mid x_{i} \in \mathbf{M}_{m(i)}^{d}, 1 \leq i \leq l, l \in \mathbf{N}\right\}
\end{gathered}
$$

We identify the element $W_{\gamma}, \gamma=\left(\gamma_{0}, \gamma_{1}, \ldots\right) \in \hat{G}_{2 \mathrm{~m}}$ with the element

$$
A_{0}^{\gamma_{0}} B_{1}^{\gamma_{1}} \otimes A_{2}^{\gamma_{2}} B_{3}^{\gamma_{3}} \otimes A_{4}^{\gamma_{4}} B_{5}^{\gamma_{5}} \cdots
$$

where for $n=0,1,2, \ldots$

$A_{2 n}=\sum_{j \in Z_{m(n)}} \exp \left(2 \pi i \frac{j}{m(k)}\right) e_{j j}^{m(n)}=\vartheta\left(\pi\left(r_{n}\right)\right)=\vartheta\left(\pi\left(0,0, \ldots, 1_{2 \mathrm{n}-\text { th place }}, 0,0, \ldots\right)\right)$

and

$$
B_{2 n+1}=\sum_{j \in \mathbf{Z}_{m(n)}} e_{j, j+1}^{m(n)}=\vartheta\left(\lambda_{\left(0,0, \ldots, 1_{(2 \mathrm{n}+1)-\text { th place }}, 0,0, \ldots\right)}\right)
$$

The following result follows now from Theorem 2.3 and Corollary 2.6. It extends results on unconditional decompositions of reflexive $L_{p}$-spaces associated with $\mathscr{R}$ from [SF1,2].

TheOrem 3.4. The system of eigenspaces

$$
\mathscr{Y}:=\left\{\delta_{n, j}\right\}_{n=0, j=1}^{\infty, m(n)-1} \cup\left\{\sigma_{n, j}\right\}_{n=0, j=1}^{\infty, m(n)-1} \cup \mathbf{1}
$$

where 


$$
\delta_{n, j}:=\left[x_{0} \otimes x_{1} \otimes \ldots \otimes x_{n-1} \otimes A_{2 n}^{j} \otimes \mathbf{1}_{m(n+1)} \otimes \ldots\right]_{x_{i} \in \mathbf{M}_{m(i)}, 1 \leq i-1}
$$

and

$$
\sigma_{n, j}:=\left[x_{0} \otimes x_{1} \otimes \ldots \otimes x_{n-1} \otimes x_{n} B_{2 n}^{j} \otimes \mathbf{1}_{m(n+1)} \otimes \ldots\right]_{x_{i} \in \mathbf{M}_{m(i)}, 1 \leq i \leq n-1, x_{n} \in \mathbf{M}_{m(n)}^{d}}
$$

forms an unconditional decomposition of any $L_{p}(\mathscr{R}, \tau)$, provided $1<p<\infty$. The same assertion holds in any symmetric operator space $E(\mathscr{R}, \tau)$ associated with $(\mathscr{R}, \tau)$ and the separable symmetric function space $E(0,1)$ with non-trivial Boyd indices.

\section{REFERENCES}

[AVDR] G. N. Agaev, N. Ya. Vilenkin, G. M. Dzafarli and A. I. Rubinštein, Multiplicative Systems and Harmonic Analysis on Zero-Dimensional Groups, Elm (1981) (Russian)

[BGM1] E. Berkson, T. A. Gillespie and P. S. Muhly, Theorie spectrale dans les espaces UMD, C. R. Acad. Sci. Paris 302 (1986), 155-158.

[BGM2] E. Berkson, T. A. Gillespie and P. S. Muhly, Generalized analyticity in UMD-spaces, Ark. Mat. 27 (1989), 1-14.

[BaR] L. A. Balashov and A. I. Rubinshtein, Series with respect to the Walsh system and their generalizations, J. Soviet Math. 1 (1973), 727-763.

[BR] O. Bratteli and D. Robinson, Operator Algebras and Quantum Statistical Mechanics I, Springer-Verlag (1979).

[CO] I. Cuculescu and A. G. Oprea, Noncommutative Probability, Mathematics and its Applications 305, Kluwer Academic Publishers, (1994).

[Di] J. Dixmier, von Neumann Algebras, North-Holland Math. Library 27 (1981).

[DDP1] P. G. Dodds, T. K. Dodds and B. de Pagter, Non-commutative Banach function spaces, Math. Z. 201 (1989), 583-597.

[DDP2] P. G. Dodds, T. K. Dodds and B. De Pagter, Fully symmetric operator spaces, Integral Equations Operator Theory 15 (1992), 942-972.

[DS] P. G. Dodds and F. A. Sukochev, Contractibility of the linear group in Banach spaces of measurable operators, Integral Equations Operator Theory 26 (1996), 305-337.

[FK] T. Fack and H. Kosaki, Generalized s-numbers of $\tau$-measurable operators, Pacific J. Math. 123 (1986), 269-300.

[FS] S. V. Ferleger and F. A. Sukochev, On the contractibilty to a point of the linear groups of reflexive non-commutative $L_{p}$-spaces, Math. Proc. Cambridge Philos. Soc. 119 (1996), 545-560.

[KPS] S. G. Krein, Ju. I. Petunin and E. M. Semenov, Interpolation of linear operators, Transl. Math. Monographs 54 (1982).

[LP] F. Lust-Piquard, Khintchine inequalities in $C_{p}(1<p<\infty)$, C. R. Acad. Sc. Paris 303 (1986), 289-292.

[LPP] F. Lust-Piquard and G. Pisier, Non-commutative Khintchine and Paley inequalities, Ark. Mat. 29 (1991), 241-260.

[LT1] J. Lindenstrauss and L. Tzafriri, Classical Banach Spaces I. Sequence Spaces, SpringerVerlag, (1977).

[LT2] J. Lindenstrauss and L. Tzafriri, Classical Banach Spaces II. Function Spaces, Springer-Verlag, (1979).

[OPT] D. Olesen, G. K. Pedersen and M. Takesaki, Ergodic actions of compact abelian groups, J. Operator Theory 3 (2) (1980), 237-271. 
[Pa] R. E. A. C. Paley, A remarkable system of orthogonal functions, Proc. Lond. Math. Soc. 34 (1932), 241-279.

[Pe] G. K. Pedersen, $C^{*}$-algebras and their automorphism groups, London Math. Soc. Monographs 14 (1979).

[PX] G. Pisier and Q. Xu, Non-commutative martingale inequalities, Comm. Math. Phys. 189 (1997), 667-698.

[Sa] S. Sakai, $C^{*}$-Algebras and $W^{*}$-Algebras, Springer-Verlag (1971).

[Se] I. E. Segal, A non-commutative extension of abstract integration, Ann. of Math. 57 (1953), 401-457.

[St] E. Størmer, Spectra of ergodic transformation, J. Funct. Anal. 15 (1974), 202-215.

[Str] S̆. Strătilă, Modular Theory in Operator Algeras, Editura Academiei and Abacus Press, (1981).

[SC] F. A. Sukochev and V. I. Chilin, Symmetric spaces on semifinite von Neumann algebras, Soviet Math. Dokl 42 (1991), 97-101.

[SF1] F. A. Sukochev and S. V. Ferleger, Harmonic analysis in symmetric spaces of measurable operators, Dokl. Akad. Nauk 339 (1994), no. 3, 307-310 (Russian).

[SF2] F. A. Sukochev and S. V. Ferleger, Harmonic analysis in UMD-spaces: application to the theory of bases, Math. Notes 58 (1995), 1315-1326.

[SWS] F. Schipp, W. R. Wade and P. Simon, Walsh Series: an Introduction to Dyadic Harmonic Analysis, Adam Hilger, (1990).

[Ta] M. Takesaki, Theory of Operator Algebras I, Springer-Verlag (1979).

[Vi] N. Ya. Vilenkin, A class of complete orthonormal systems, Izv. Akad. Nauk SSSR, Ser. Mat 11 (1947), 363-400 (Russian); Amer. Math. Soc. Transl. Ser. 228 (1963), 135 .

[Wa] C. Watari, On generalized Walsh-Fourier series, Tôhoku Math J. (2) 10 (1958), 211241.

DEPARTMENT OF MATHEMATICS AND STATISTICS

THE FLINDERS UNIVERSITY OF SOUTH AUSTRALIA

GPO BOX 2100

ADELAIDE, SA 5001

AUSTRALIA

E-mail: peter@ist.flinders.edu.au, sukochev@ist.flinders.edu.au 